\title{
Preoperative Neutrophil to Lymphocyte Ratio as a Prognostic Factor in Patients with Non-metastatic Renal Cell Carcinoma
}

\author{
Ru-Min Wen ${ }^{1,2 \&}$, Yi-Jing Zhang ${ }^{1,2 \&}$, Sha $\mathrm{Ma}^{3}$, Ying-Li Xu ${ }^{4}$, Yan-Su Chen ${ }^{5}$, Hai- \\ Long Li ${ }^{1,2}$, Jin Bai ${ }^{1 *}$, Jun-Nian Zheng ${ }^{1 *}$
}

\begin{abstract}
Background: The neutrophil-to-lymphocyte ratio (NLR) is a strong predictor of mortality in patients with colorectal, lung, gastric cancer, pancreatic and metastatic renal cell carcinoma. We here evaluated whether preoperative NLR is an independent prognostic factor for non-metastatic renal cell carcinoma (RCC). Materials and Methods: Data from 327 patients who underwent curative or palliative nephrectomy were evaluated retrospectively. In preoperative blood routine examination, neutrophils and lymphocytes were obtained. The predictive value of NLR for non-metastatic RCC was analyzed. Results: The NLR of 327 patients was 2.72 \pm 2.25 . NLR $<1.7$ and NLR $\geq 1.7$ were classified as low and high NLR groups, respectively. Chi-square test showed that the preoperative NLR was significantly correlated with the tumor size $(P=0.025)$, but not with the histological subtype $(P=0.095)$ and the $p T$ stage $(P=0.283)$. Overall survival $(O S)$ and disease-free survival $(D F S)$ were assessed using the Kaplan-Meier method. Effects of NLR on OS $(P=0.007)$ and DFS $(P=0.011)$ were significant. To evaluate the independent prognostic significance of NLR, multivariate COX regression models were applied and identified increased NLR as an independent prognostic factor for $O S(P=0.015)$, and DFS $(P=0.019)$. Conclusions: Regarding patient survival, an increased NLR represented an independent risk factor, which might reflect a higher risk for severe cardiovascular and other comorbidities. An elevated blood NLR may be a biomarker of poor OS and DFS in patients with non-metastatic RCC.
\end{abstract}

Keywords: Non-metastatic renal cell carcinoma - neutrophil-to-lymphocyte ratio - overall survival - disease-free survival

Asian Pac J Cancer Prev, 16 (9), 3703-3708

\section{Introduction}

Renal cell carcinoma is the most common malignant tumor of the kidney, accounting for about $3 \%$ of malignancies in adults (Siegel et al., 2013). Thanks to an increased use of ultrasound and computed tomography in recent years, incidental and early stage tumors disease have been encountered more frequently (Kim et al., 2011). Although the increase in the number of early stage tumors, the disease mortality rate is still increasing (Kim et al., 2011). Most of these cancers are localized in the kidney, but after nephrectomy metastasis develops in $20 \%$ to $30 \%$ of patients with localized disease (Roos et al., 2014). As renal tumors are unresponsive to chemotherapy and radiotherapy, the majority of metastatic patients die (Grunwald and Ravaud, 2014). Although TNM stage and nuclear grade are currently widely accepted as prognostic factors, they are not entirely reliable (Shuch et al., 2006). Other well-known prognostic factors are surgical margin positivity, lymphovascular invasion and histological subtype. Due to the insufficiency of these prognostic factors, new factors including clinical and laboratory findings have started to be studied.
Increasing evidence supports the involvement of systemic inflammation in cancer development and progression (Mantovani et al., 2008). It has been shown that, among the leukocytes in circulation, neutrophils increase and lymphocytes decrease relatively as a systemic inflammatory response development against the tumor. The NLR has been used as an indicator of systemic inflammatory response (Zahorec, 2001). It was reported that increased pretreatment NLR is associated with poor outcome in colorectal (Walsh et al., 2005), gastric (Ubukata et al., 2010) and ovarian (Cho et al., 2009) cancer cases. Cumulating evidence in metastatic RCC suggests that a high NLR might represent an independent adverse prognostic factor in interferon-treated (Atzpodien et al., 2003), interleukin-2 treated (Donskov and von der Maase, 2006), as well as in sunitinib-treated (Keizman et al., 2012) patients. However, in non-metastatic RCC, data regarding the prognostic significance of the NLR are sparse, and controversy still exists about how discriminating the NLR might potentially be an independent risk factor. Based on these, the aim of our study was to further clarify the prognostic significance of the preoperative NLR in nonmetastatic RCC and to evaluate whether this parameter

${ }^{1}$ Jiangsu Center for the Collaboration and Innovation of Cancer Biotherapy, Cancer Institute, ${ }^{2}$ Department of Urology, ${ }^{3}$ Department of Hematology, ${ }^{4}$ Department of Operation, the Affiliated Hospital of Xuzhou Medical College, ${ }^{5}$ Department of Public health, Xuzhou Medical College,Xuzhou, China ${ }^{\circledR}$ Equal contributors*For correspondence: bj@xzmc.edu.cn, jnzheng@xzmc.edu.cn 
provides additional prognostic information to wellestablished clinicopathological parameters.

\section{Materials and Methods}

\section{Basic information of patients}

This retrospective analysis included data from 361 consecutive NO RCC patients who underwent a curative radical or partial nephrectomy at the Department of Urology in the Affiliated Hospital of Xuzhou Medical College, China, between December 2005 and December 2011. All of the clinicopathological data were retrieved from pathology reports from the Department of Pathology at the same hospital. Of this data set, we excluded 29 cases with NOMx RCC, and 5 cases who had hematologic disease, metastatic disease, active infection, and received neoadjuvant therapy (Eryilmaz et al., 2014) that may affect the number of leukocytes during diagnosis, were excluded from the study. The remaining 327 patients with non-metastatic RCC $\left(\mathrm{T}_{1}-\mathrm{T}_{4} \mathrm{~N}_{0} \mathrm{M}_{0}\right)$ were included into the study. The laboratory data, including neutrophil and lymphocyte counts, were obtained by preoperative exploration one day before surgical intervention. The NLR was calculated by dividing the number of absolute neutrophils by the number of lymphocytes.

\section{Follow-up of patients}

Patients' postoperative surveillance included routine clinical and laboratory examination; regarding imaging methods, X-rays of the chest and abdominal ultrasound were predominantly used, especially in patients with a low relapse risk $\left(\mathrm{pT}_{1}\right)$, whereas computed tomography or magnetic resonance imaging was performed in all other patients as Pichler et al. (2011) previously reported. Follow-up evaluations were performed every 6 months for the first 5 years and annually thereafter for locally advanced tumors. In organ-confined cancers, imaging was performed twice in the first year after surgery and annually thereafter. No neoadjuvant or adjuvant treatment was administered. OS was defined as the time (in months) from date of surgery to individuals' death for any cause. DFS was defined as the time (in months) from date of surgery to non-cancer related death. Recurrence was defined by every new occurrence of kidney cancer after nephrectomy, and local, metastatic recurrence or death from kidney cancer. During the follow-up, when patients are still alive at the last or lost in the process, the data were identified as censored. The study was approved by the local ethical committee of the Xuzhou Medical College.

\section{Statistical analysis}

Data are expressed as the mean \pm SD. The study end point was OS and DFS. Factors analyzed were patient age, gender, the histological subtypes, pT stage, neutrophil count, lymphocyte count and NLR. Categorical data independence of fit was analyzed by the chi-square test. As reported previously (Atzpodien et al., 2003), continuous variables such as age, neutrophil count, lymphocyte count and NLR were analyzed as dichotomous variables according to approximate optimal cutoff points. The NLR cutoff value that best discriminated between good and poor survival, that is with the most significant $\mathrm{P}$ value according to the log rank test, was determined by testing all possible cutoffs by ROC. And the median value of age, neutrophil count, lymphocyte count was the cutoff value. All such cutoffs were then rounded to clinically relevant (convenient) values.

The relationship between NLR and other clinicopathologic parameters was studied by nonparametric tests. Patients' clinical end points were calculated using the Kaplan-Meier method and compared by the log rank test. Enter stepwise multivariate COX proportion analysis was performed to determine the influence of pT stage, age, gender, the histological subtypes, and NLR on DFS and OS. Hazard ratios (HRs) estimated from the Cox analysis were reported as relative risks with corresponding 95\% confidence intervals (CI). The best cutoff value of the NLR at survival time was tested by using the STATA 10.0. All other statistical analyses were performed using the SPSS 19.0 (SPSS Inc., Chicago, IL, USA). A two-sided $P<0.05$ was considered statistically significant.

\section{Results}

Clinicopathologic characteristics of our study cohort As shown in Table 1, of the 327 consecutive RCC patients, 294 (89.9\%) had clear cell, 11 (3.4\%) had

Table 1. Clinical, Surgical, And Pathologic Features of 327 Patients Who Underwent Nephrectomy for NonMetastatic RCC

\begin{tabular}{|c|c|c|}
\hline \multicolumn{2}{|c|}{ Features } & \multirow{2}{*}{$\frac{\text { Value }}{56.15 \pm 12.727}$} \\
\hline Age (years) & Mean \pm SD & \\
\hline & Median & 56 \\
\hline & Range & $3-86$ \\
\hline \multirow{2}{*}{ No. gender $(\%)$} & Male & $211(64.5)$ \\
\hline & Female & $116(35.5)$ \\
\hline \multirow[t]{6}{*}{ No. histology $(\%)$} & Conventional & $294(89.9)$ \\
\hline & Chromophobe & $5(1.5)$ \\
\hline & Papillary & $11(3.4)$ \\
\hline & Multilocular cystic & $2(0.6)$ \\
\hline & Adenocarcinoma & $10(3.1)$ \\
\hline & Others & $5(1.5)$ \\
\hline \multirow[t]{4}{*}{ pT stage ${ }^{a}(\%)$} & $\mathrm{T}_{1}$ & $236(72.2)$ \\
\hline & $\mathrm{T}_{2}$ & $50(15.3)$ \\
\hline & $\mathrm{T}_{3}^{2}$ & $40(12.2)$ \\
\hline & $\mathrm{T}_{4}^{3}$ & $1(0.3)$ \\
\hline \multirow[t]{3}{*}{ Neutrophils } & Mean \pm SD & $4309 \pm 2045$ \\
\hline & Median & 3840 \\
\hline & Range & $1160-15660$ \\
\hline \multirow[t]{3}{*}{ Lymphocytes } & Mean \pm SD & $1791 \pm 546$ \\
\hline & Median & 1740 \\
\hline & Range & $450-3500$ \\
\hline \multirow[t]{5}{*}{ NLR } & Mean \pm SD & $2.72 \pm 2.25$ \\
\hline & Median & 2.21 \\
\hline & Range & $0.77-25.69$ \\
\hline & First quartile & 1.7 \\
\hline & Third quartile & 2.9 \\
\hline \multirow[t]{2}{*}{ Tumor size (\%) } & $>7 \mathrm{~cm}$ & $62(19.0)$ \\
\hline & $\leq 7 \mathrm{~cm}$ & $265(81.0)$ \\
\hline
\end{tabular}

*SD: standard deviation; according to the 2010 American Joint Committee on Cancer TNM staging system 
Preoperative Neutrophil to Lymphocyte Ratio as a Prognostic Factor in Patients with Non-Metastatic Renal Cell Carcinoma

papillary, 5 (1.5\%) had chromophobe, $2(0.6 \%)$ had multilocular cystic, $10(3.1 \%)$ had adenocarcinoma, 5 $(1.5 \%)$ were not otherwise specified. In the $211(64.5 \%)$ men and $116(35.5 \%)$ women, median age at nephrectomy was 56 years (range 3 to 86). The pT stage was T1 to T4 in $236(72.2 \%), 50(15.3 \%), 40(12.2 \%), 1(0.3 \%)$ cases, respectively. Mean laboratory values were neutrophil count $4,309 \pm 2,045 / \mu 1$, lymphocyte count $1,791 \pm 546 / \mu \mathrm{l}$ and NLR 2.72 \pm 2.25 . According to tumor size, the patients were collected as two groups: $>7 \mathrm{~cm}$ was $62(19.0 \%)$, and $\leq 7 \mathrm{~cm}$ was $265(81.0 \%)$. Mean follow-up was 43 months (range 0 to 97). During follow-up disease recurred in 22

Table 2. The Relationship between Patients' Preoperative Neutrophil-To-Lymphocyte Ratio (NLR) and Age, Gender, Tumor Sizes, the Histologic Subtypes, Pt Stage Results Compared by Chi-Square Test

\begin{tabular}{lcrrrr}
\hline & \multicolumn{5}{c}{ Preoperative NLR } \\
\cline { 2 - 6 } Variables & $<1.7(\%)$ & $\geq 1.7(\%)$ & Total & $\chi^{2}$ & $\mathrm{P}^{*}$ \\
\hline All cases & $78(23.9)$ & $249(76.1)$ & 327 & & \\
Age (years) & & & & & \\
$\quad<56$ & $37(47.4)$ & $118(47.4)$ & 155 & 0.000 & 0.994 \\
$\geq 56$ & $41(52.6)$ & $131(52.6)$ & 172 & & \\
Gender & & & & & \\
$\quad$ Male & $44(56.4)$ & $167(67.1)$ & 211 & 2.948 & 0.086 \\
Female & $34(43.6)$ & $82(32.9)$ & 116 & & \\
Tumor size & & & & & \\
$\leq 7$ cm & $70(89.7)$ & $195(78.3)$ & 265 & 5.05 & 0.025 \\
$>7$ cm & $8(10.3)$ & $54(21.7)$ & 62 & & \\
Histologic subtypes & & & & \\
$\quad$ Clear cell & $74(94.9)$ & $220(88.4)$ & 294 & 2.781 & 0.095 \\
$\quad$ Non-clear cell & $4(5.1)$ & $29(11.6)$ & 33 & & \\
pT stage & & & & & \\
$\mathrm{T}_{1}$ & $60(76.9)$ & $176(70.7)$ & 236 & 1.152 & 0.283 \\
$\mathrm{~T}_{2}-\mathrm{T}_{4}$ & $18(23.1)$ & $73(29.3)$ & 91 & & \\
\hline *Two sided Fisher's exact tests & & & & \\
\hline \multicolumn{5}{r}{} \\
\hline
\end{tabular}
patients at a mean of 30 months (range 3 to 56). The site of recurrence was lung in 6 cases, liver in 1 , brain in 1 , renal in 7 , and other in 7.

\section{NLR was significantly associated with tumor size}

According to the ROC curve (Figure 1), we determined the cutoff of 1.7 for the NLR to be optimal to discriminate patients' survival that prompted us to select 1.7 as the optimal cutoff value for all subsequent analyses to differentiate between low $(<1.7)$ and high $(\geq 1.7)$ NLR. Overall, there are $78(23.9 \%)$ patients with a low NLR and $249(76.1 \%)$ patients with a high NLR. As shown in Table 2, chi-square analysis revealed that the increased preoperative NLR in the non-metastatic RCC was significantly positively associated with tumor size

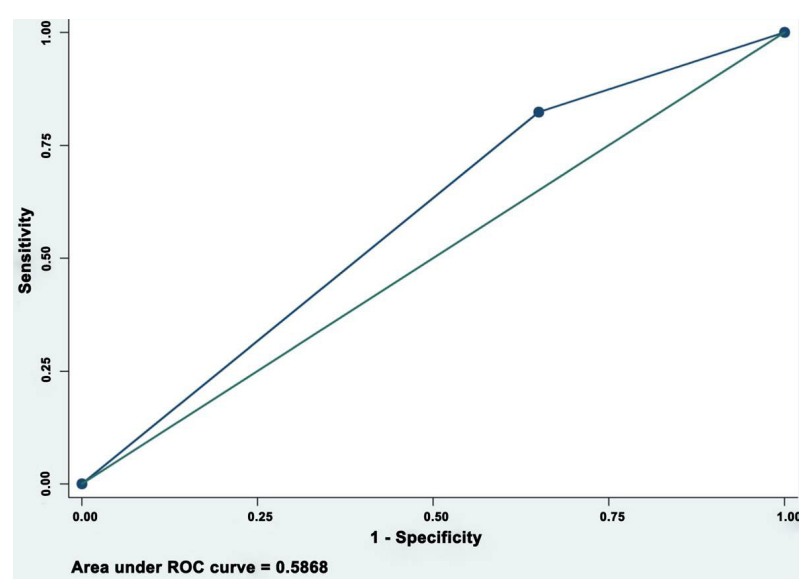

Figure 1. The NLR Cutoff Value that Best Discriminated Between Good and Poor Survival, That is with the most significant $P$ value according to the log rank test, was determined by testing all possible cutoffs by ROC. The area under the curve (AUC) at cutoff value 1.7 of the NLR at survival time was calculated

Table 3. Univariate Cox Proportional Regression Analysis on Overall Survival and Disease-Free Survival of 327 Non-Metastatic Rec Patients

\begin{tabular}{lccccccc}
\hline \multirow{2}{*}{ Group } & \multicolumn{3}{c}{ Overall survival } & & \multicolumn{3}{c}{ Disease-free survival } \\
\cline { 2 - 4 } \cline { 5 - 7 } & HR & $95 \% \mathrm{CI}^{\dagger}$ & $P^{*}$ & & HR & $95 \% \mathrm{CI}^{\dagger}$ & $P^{*}$ \\
\hline Gender (Male $v s$ Female) & 1.123 & $0.809-1.561$ & 0.488 & & 1.157 & $0.812-1.648$ & 0.419 \\
Age $(\geq 56$ years $v s<56$ years) & 1.126 & $0.820-1.547$ & 0.463 & & 0.968 & $0.688-1.362$ & 0.852 \\
The histologic subtypes & 1.486 & $0.928-2.379$ & 0.099 & & 1.425 & $0.844-2.406$ & 0.185 \\
(Clear cell $v s$ Non-clear cell) & & & & & & \\
pT stage $\left(\mathrm{T}_{2}-\mathrm{T}_{4} v s \mathrm{~T}_{1}\right)$ & 1.476 & $1.050-2.074$ & 0.025 & & 1.33 & $0.912-1.940$ & 0.139 \\
NLR $(\geq 1.7 v s<1.7)$ & 1.746 & $1.158-2.632$ & 0.008 & & 1.756 & $1.127-2.736$ & 0.013 \\
Tumor size $(>7 \mathrm{~cm} v s \leq 7 \mathrm{~cm})$ & 1.93 & $1.348-2.762$ & 0.000 & & 1.757 & $1.174-2.629$ & 0.006 \\
\hline
\end{tabular}

$* P$ values are from Log-rank test; ${ }^{\dagger} \mathrm{CI}$ : confidence interval

Table 4. Multivariate Cox Regression Analysis on Overall Survival and Disease-Free Survival of 327 NonMetastatic Rcc Patients

\begin{tabular}{llcccccc}
\hline Group & \multicolumn{3}{c}{ Overall survival } & & \multicolumn{3}{c}{ Disease-free survival } \\
\cline { 2 - 4 } & $\mathrm{HR}$ & $95 \% \mathrm{CI}^{\dagger}$ & $P^{*}$ & & $\mathrm{HR}$ & $95 \% \mathrm{CI}^{\dagger}$ & $P^{*}$ \\
\hline Gender (Male $v s$ Female) & 1.188 & $0.853-1.654$ & 0.309 & & 1.222 & $0.856-1.744$ & 0.271 \\
Age $(\geq 56$ years $v s<56$ years) & 1.13 & $0.821-1.554$ & 0.453 & & 0.968 & $0.686-1.365$ & 0.852 \\
The histologic subtypes & 1.405 & $0.874-2.258$ & 0.161 & & 1.293 & $0.761-2.197$ & 0.341 \\
(Clear cell $v s$ Non-clear cell) & & & & & & \\
pT stage $\left(\mathrm{T}_{2}-\mathrm{T}_{4} v s \mathrm{~T}_{1}\right)$ & 1.398 & $0.992-1.969$ & 0.056 & & 1.264 & $0.864-1.849$ & 0.228 \\
NLR $(\geq 1.7 v s<1.7)$ & 1.674 & $1.103-2.539$ & 0.015 & & 1.714 & $1.092-2.691$ & 0.019 \\
\hline
\end{tabular}

$* P$ values are from Log-rank test; ${ }^{\dagger} \mathrm{CI}$ : confidence interval 
Ru-Min Wen et al
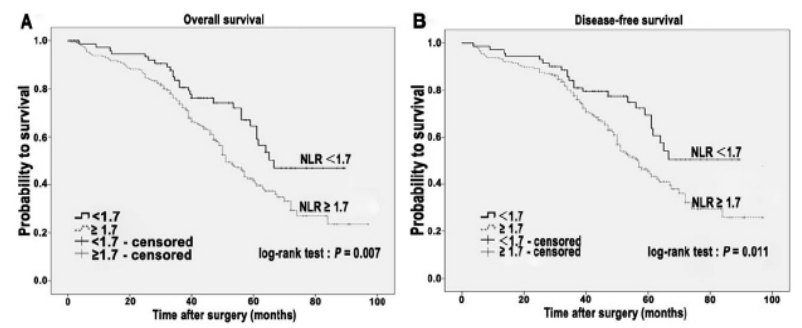

Figure 2. Kaplan-Meier Survival Analyses of 327 Non-

Metastatic RCC Patients. A Low NLR $(<1.7)$ correlates with an improved overall survival $(P=0.007, \log$-rank test). B Low NLR $(<1.7)$ correlates with an improved disease-free survival $(P=0.011$, log-rank test $)$. Cum, cumulative

$(P=0.025)$. However, we did not find significant correlate between NLR with other clinicopathologic features, including age, gender, pT stage, and the histological subtypes.

\section{Increased NLR correlates with poor patient survival}

To further investigate whether increased NLR in nonmetastatic RCC correlates with a worse prognosis, KaplanMeier survival curves were constructed using overall or disease-free cumulative survival to compare the patients with high NLR to those with low NLR ( $n=327$, follow-up time (months), median 44, range from 0 to 97 ). Our data revealed that high NLR correlated with both worse overall and disease-free survival in non-metastatic RCC $(P=0.007$ and $P=0.011$, respectively, log-rank test; Figure 2).

$N L R$ serves as an independent prognostic indicator for non-metastatic RCC

Moreover, we examined whether NLR was an independent prognostic factor for non-metastatic RCC. We performed a univariate Cox regression analysis including NLR, age, gender, pT stage, histological subtypes and tumor size to study the effects NLR on patient survival in non-metastatic RCC. The univariate Cox regression analysis showed that NLR was an independent prognostic marker for non-metastatic RCC patients overall survival (hazard ratio, 1.746; 95\% CI, 1.158-2.632; $P=0.008$; Table 3) and disease-free survival (hazard ratio, 1.756; 95\% CI, 1.127-2.736; $P=0.013$; Table 3 ). In multivariate Cox regression analysis, we found that NLR was an independent prognostic marker for overall survival (hazard ratio, 1.674; 95\% CI, 1.103-2.539; $P=0.015$; Table 4 ) and disease-free survival (hazard ratio, 1.714 ; $95 \%$ CI, 1.092 2.691; $P=0.019$; Table 4 ) after adjusting with age, gender, and histological subtypes in non-metastatic RCC.

\section{Discussion}

Despite recent progress in the identification of genetic, epigenetic and common molecular alterations in RCC has been made (Gerlinger et al., 2012), the routine diagnostic and prognostic assessment of RCC currently relies on pathological tissue examination and traditional clinicopathological prognostic variables (Ficarra et al., 2010). The complexity of these molecular changes, as well as high costs of analyses, the time-consuming preparation required and the lack of evidence demonstrating how these newly discovered molecular markers influence diagnostic or therapeutic decisions have rendered none of the markers available for routine testing.

Increasing evidence supports the involvement of systemic inflammation in cancer development and progression (Mantovani et al., 2008). Regularly used bloodbased parameters, such as the neutrophils or lymphocyte count and the resultant-derived NLR, are relatively easy to assess without additional laborious efforts, making them attractive parameters for patients' improved individualized risk assessment in RCC (Zahorec, 2001). An elevated NLR reflects both a decreased lymphocyte mediated antitumor immune response and a heightened neutrophil dependent inflammatory reaction. Both of these factors may contribute to aggressive tumor biology, cancer progression, and poor prognosis (Proctor et al., 2011; Ishizuka et al., 2013). Circulating neutrophils have been shown to produce cytokines, such as tumor necrosis factor, IL-1, and IL-6, and to secrete the proangiogenic factor VEGF (Cho et al., 2009). In addition, a relative lymphocytopenia may reflect a lower count of CD4+ Th-lymphocytes, resulting in a suboptimal lymphocytemediated immune response to malignancy. In conclusion, the NLR may reflect the combined prognostic information of these two processes and be a stronger predictor of the outcome than either of them considered alone.

An elevated pretreatment NLR has been previously demonstrated as a poor prognostic factor for different human cancer types, including gastrointestinal, soft tissue sarcoma, nasopharyngeal, as well as lung cancer (Roxburgh and McMillan, 2010). In non-metastatic RCC, only a few numbers of studies have been published about the prognostic value of the pretreatment NLR so far, and the reported findings are conflicting. In our study, we found that an increased NLR was an independent predictor for OS and DFS in a cohort of 327 non-metastatic RCC patients in China. In a validation study that included a large European cohort of 678 patients with non-metastatic clear cell RCC (Pichler et al., 2011), they demonstrated that an increased NLR was an independent negative predictor for patients' OS, but not a predictor for direct cancer related end points, such as CSS and MFS. Ohno et al (Ohno et al., 2010) reported that an increased NLR was an independent predictor for relapse-free survival in a cohort of 192 non-metastatic RCC patients from Japan. However, a definitive explanation for these observations remains speculative. Nevertheless, several studies have clearly established an increased NLR as a negative prognostic predictor for cardiovascular events (Gibson et al., 2007; Tsai et al., 2007), hypertension and diabetes (Imtiaz et al., 2012), bacteraemia (Terradas et al., 2012), chronic kidney disease (Sun et al., 2012), and severe gastrointestinal conditions (Azab et al., 2011; Alkhouri et al., 2012). In conclusion, an increased NLR seems to be associated with patients' increased risk of cardiovascular, infectious, and gastrointestinal morbidity and mortality.

To accurate predict the long-term survival immediately after nephrectomy, a prognosis model for counseling, scheduling followup visits and identifying poor risk cases potentially appropriate for adjuvant therapy protocols is needed. As for the prognosis model, Pichler $M$ et al (Pichler et al., 2011) analyzed the addition of the NLR 
Preoperative Neutrophil to Lymphocyte Ratio as a Prognostic Factor in Patients with Non-Metastatic Renal Cell Carcinoma

to the Leibovich prognosis model (Leibovich et al., 2003), which limits the analysis to patients with clear cell RCC. Another study by Kattan et al (Kattan et al., 2001) reported a postoperative nomogram for predicting treatment failure (clinical evidence of recurrence or death from RCC). In this nomogram, predictor variables were patient symptoms, histology, tumor size, and pT stage $\left(\mathrm{T}_{1}\right.$ $\mathrm{T}_{3 \mathrm{~b} / \mathrm{c}} \mathrm{N}_{0} \mathrm{M}_{0}$ ). Cindolo et al (Cindolo et al., 2003) developed a model for the preoperative prediction of diseasefree survival for non-metastatic RCC. The recurrence risk formula was constructed as $(1.28 \times$ presentation $($ asymptomatic $=0$; symptomatic $=1)+(0.13 \times$ clinical size $)$. And patients with tumor extension beyond Gerota's fascia $\left(\mathrm{pT}_{4}\right)$ were excluded from the study. But in our study, the tumor sizes of patients were not continuous variables. Ohno et al. (2010) developed a preoperative risk stratification model for recurrence based on pT stage as a tumor related factor and NLR as a host related factor. In their study, they retrospectively reviewed the records of 192 patients with non-metastatic RCC $\left(\mathrm{T}_{1}{ }_{4} \mathrm{~N}_{0} \mathrm{M}_{0}\right)$, using the two statistically significant variables (pT stage and NLR) obtained from multivariate analysis for RFS. The site of recurrence was lung in 20 cases, bone in 7 , liver in 3, brain in 2, retroperitoneal space in 2 and other in 9. But in our study, the study end point was OS and DFS. And for OS and DFS, pT stage is not a statistically significant variable. So, there is not a suitable prognosis model for our study.

In our study, we observed that the NLR is only associated with tumor volume, which is consistent with Ceylan C et al (Ceylan et al., 2014) reported. The reason why pT stage is not a statistically significant variable for OS and DFS remains speculative. Although the prognostic value of $\mathrm{pT}$ stage is well established in a vast number of studies, as we all know, it is also known that tumors with similar pathological stage do not always exhibit similar biological behavior and aggressiveness. And the definition of the study endpoint of OS and DFS, and the

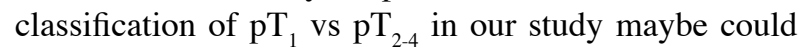
explain it. As with all retrospective studies, limitations of our study are inherent to its design including the retrospective data collection. Although the NLR is easy to measure, conditions such as active infection, inflammatory diseases, smoking behavior or stress at the time of blood collection may affect it (Pedersen et al., 1999). Nonetheless, even considering these limitations, our data indicate that an increased pretreatment NLR might represent an independent prognostic factor for OS and DFS in non-metastatic RCC patients, although further validation is required.

\section{Acknowledgements}

This project is supported by grants from the National Natural Science Foundation of China (No.81472663, 81201636), and China Postdoctoral Science Foundation (No. 2014T70549, 2012M511323).

\section{References}

Alkhouri N, Morris-Stiff G, Campbell C, et al (2012).
Neutrophil to lymphocyte ratio: a new marker for predicting steatohepatitis and fibrosis in patients with nonalcoholic fatty liver disease. Liver Int, 32, 297-302.

Atzpodien J, Royston P, Wandert T, et al (2003). Metastatic renal carcinoma comprehensive prognostic system. $\mathrm{Br} J$ Cancer, 88, 348-53.

Azab B, Jaglall N, Atallah JP, et al (2011). Neutrophillymphocyte ratio as a predictor of adverse outcomes of acute pancreatitis. Pancreatology, 11, 445-52.

Ceylan C, Camtosun A, Doluoglu OG, et al (2014). Emphasis of neutrophil-to-lymphocyte ratio in non-metastatic renal cell carcinoma. Urologia, 81, 51-6.

Cho H, Hur HW, Kim SW, et al (2009). Pre-treatment neutrophil to lymphocyte ratio is elevated in epithelial ovarian cancer and predicts survival after treatment. Cancer Immunol Immunother, 58, 15-23.

Cindolo L, de la Taille A, Messina G, et al (2003). A preoperative clinical prognostic model for non-metastatic renal cell carcinoma. BJU Int, 92, 901-5.

Donskov F, von der Maase H (2006). Impact of immune parameters on long-term survival in metastatic renal cell carcinoma. J Clin Oncol, 24, 1997-2005.

Eryilmaz MK, Mutlu H, Salim DK, et al (2014). The neutrophil to lymphocyte ratio has a high negative predictive value for pathologic complete response in locally advanced breast cancer patients receiving neoadjuvant chemotherapy. Asian Pac J Cancer Prev, 15, 7737-40.

Ficarra V, Brunelli M, Cheng L, et al (2010). Prognostic and therapeutic impact of the histopathologic definition of parenchymal epithelial renal tumors. Eur Urol, 58, 655-68.

Gerlinger M, Rowan AJ, Horswell S, et al (2012). Intratumor heterogeneity and branched evolution revealed by multiregion sequencing. $N$ Engl J Med, 366, 883-92.

Gibson PH, Croal BL, Cuthbertson BH, et al (2007). Preoperative neutrophil-lymphocyte ratio and outcome from coronary artery bypass grafting. Am Heart J, 154, 995-1002.

Grunwald V, Ravaud A (2014). Systemic therapy of renal cell carcinoma. World J Urol, 32, 1.

Imtiaz F, Shafique K, Mirza SS, et al (2012). Neutrophil lymphocyte ratio as a measure of systemic inflammation in prevalent chronic diseases in Asian population. Int Arch Med, 5, 2 .

Ishizuka M, Nagata H, Takagi K, et al (2013). Combination of platelet count and neutrophil to lymphocyte ratio is a useful predictor of postoperative survival in patients with colorectal cancer. Br J Cancer, 109, 401-7.

Kattan MW, Reuter V, Motzer RJ, et al (2001). A postoperative prognostic nomogram for renal cell carcinoma. J Urol, 166, 63-7.

Keizman D, Ish-Shalom M, Huang P, et al (2012). The association of pre-treatment neutrophil to lymphocyte ratio with response rate, progression free survival and overall survival of patients treated with sunitinib for metastatic renal cell carcinoma. Eur J Cancer, 48, 202-8.

Kim SP, Shah ND, Weight CJ, et al (2011). Contemporary trends in nephrectomy for renal cell carcinoma in the United States: results from a population based cohort. J Urol, 186, 1779-85.

Leibovich BC, Blute ML, Cheville JC, et al (2003). Prediction of progression after radical nephrectomy for patients with clear cell renal cell carcinoma: a stratification tool for prospective clinical trials. Cancer, 97, 1663-71.

Mantovani A, Allavena P, Sica A, et al (2008). Cancer-related inflammation. Nature, 454, 436-44.

Ohno Y, Nakashima J, Ohori M, et al (2010). Pretreatment neutrophil-to-lymphocyte ratio as an independent predictor of recurrence in patients with nonmetastatic renal cell carcinoma. J Urol, 184, 873-8. 
Ru-Min Wen et al

Pedersen BK, Bruunsgaard H, Jensen M, et al (1999). Exercise and immune function: effect of ageing and nutrition. Proc Nutr Soc, 58, 733-42.

Pichler M, Hutterer GC, Chromecki TF, et al (2011). External validation of the Leibovich prognosis score for nonmetastatic clear cell renal cell carcinoma at a single European center applying routine pathology. J Urol, 186, 1773-7.

Proctor MJ, Morrison DS, Talwar D, et al (2011). A comparison of inflammation-based prognostic scores in patients with cancer. a glasgow inflammation outcome study. Eur $J$ Cancer, 47, 2633-41.

Roos FC, Steffens S, Junker K, et al (2014). Survival advantage of partial over radical nephrectomy in patients presenting with localized renal cell carcinoma. BMC Cancer, 14, 372 .

Roxburgh CS, McMillan DC (2010). Role of systemic inflammatory response in predicting survival in patients with primary operable cancer. Future Oncol, 6, 149-63.

Shuch BM, Lam JS, Belldegrun AS, et al (2006). Prognostic factors in renal cell carcinoma. Semin Oncol, 33, 563-75.

Siegel R, Naishadham D, Jemal A (2013). Cancer statistics, 2013. CA Cancer J Clin, 63, 11-30.

Sun M, Bianchi M, Hansen J, et al (2012). Chronic kidney disease after nephrectomy in patients with small renal masses: a retrospective observational analysis. Eur Urol, 62, 696-703.

Terradas R, Grau S, Blanch J, et al (2012). Eosinophil count and neutrophil-lymphocyte count ratio as prognostic markers in patients with bacteremia: a retrospective cohort study. PLoS One, 7, 42860 .

Tsai JC, Sheu SH, Chiu HC, et al (2007). Association of peripheral total and differential leukocyte counts with metabolic syndrome and risk of ischemic cardiovascular diseases in patients with type 2 diabetes mellitus. Diabetes Metab Res Rev, 23, 111-8.

Ubukata H, Motohashi G, Tabuchi T, et al (2010). Evaluations of interferon-gamma/interleukin-4 ratio and neutrophil/ lymphocyte ratio as prognostic indicators in gastric cancer patients. J Surg Oncol, 102, 742-7.

Walsh SR, Cook EJ, Goulder F, et al (2005). Neutrophillymphocyte ratio as a prognostic factor in colorectal cancer. J Surg Oncol, 91, 181-4.

Zahorec R (2001). Ratio of neutrophil to lymphocyte countsrapid and simple parameter of systemic inflammation and stress in critically ill. Bratisl Lek Listy, 102, 5-14. 